NASA Technical Memorandum 103151

\title{
Automated Electric Power Management and Control for Space Station Freedom
}

James L. Dolce, Pamela A. Mellor, and James A. Kish

Lewis Research Center

Cleveland, Ohio

(NASA-TM-103151) AUTOMATED ELECTPIC PUHER MANAGFMENT ANO CONTROL FOR SPACE STATION

FREEDTM (NASA) a D
CSCL $09 B$
N90-23125

Unclas

0201589

Prepared for the

25th Intersociety Energy Conversion Engineering Conference cosponsored by the AIChE, SAE, ACS, AIAA, ASME, and IEEE Reno, Nevada, August 12-17, 1990 
$\rightarrow-$ 


\title{
Automated Electric Power Management and Control for Space Station Freedom
}

\author{
James L. Dolce, Pamela A. Mellor, and James A. Kish \\ National Aeronautics and Space Administration \\ Lewis Research Center \\ Cleveland, Ohio 44135
}

\begin{abstract}
A comprehensive automation design is being developed for Space Station Freedom's electric power system. A joint effort between NASA's Office of Aeronautics and Space Technology and NASA's Office of Space Station Freedom, it strives to increase station productivity by applying expert systems and conventional algorithms to automate power system operation. An integrated approach to the power system command and control problem is defined and used to direct technology development in: diagnosis, security monitoring and analysis, battery management, and cooperative problem-solving for resource allocation. The prototype automated power system is developed using simulations and test-beds.
\end{abstract}

\section{Introduction}

The paramount objective for our power system's operation is: to generate and dispatch electric power to the loads while maximizing Space Station Freedom's productivity and without violating any constraints. The initial station operation will use dispatchers aided by human-interactive computational facilities to perform the necessary command and control tasks. These tasks constitute planning and decision-making activities that strive to eliminate unplanned outages. To make quality decisions, the dispatchers must have an acumen sharpened through years of experience. Space Station Freedom will adopt an intensive human command and control approach initially, but we perceive that in the long run there are opportunities to re- duce our reliance upon skilled dispatchers and to make faster and more consistent on-line decisions by capturing this knowledge in expert systems [1].

\section{Power System Automation}

\subsection{Perspective}

The Space Station Freedom's electric power system is a complex subsystem which must be operated in concert with other station subsystems and payloads. Using automation to decrease the human involvement in these operating activities will increase productivity.

Freedom's operation itself can be viewed as two interactive tasks: apportioning the available resources among the subsystems and payloads and operating those subsystems and payloads. The apportioning tasks must insure that station productivity is maximized while the operating tasks insure that station integrity is not jeopardized. In the case of the electric power system, the apportioning of electric energy divides among storage and loads. This activity must reserve adequate energy for periods of eclipse, maximize experiment productivity, and respond to emergency power losses [2].

Within the electric power system, resource apportioning and system operation can be accomplished using a recognize-act procedure. The recognition part acquires data and then classifies the quality of the operating state and its performance status using subsystem functional knowledge and diagnostic ability. The action part of the procedure apportions the electric resource and dispatches it through the distribu- 
tion network using value judgment and apportioning knowledge. The recognize-act procedure is repeated whenever new data requires new action. This procedural paradigm requires technology development and demonstration in five areas of knowledge [1]:

- Detection and Classification: This area applies discriminative knowledge to convert quantitative state data into qualitative classifications of operating behavior.

- Diagnosis: This area applies diagnostic knowledge to determine the cause of failures.

- Forecasting: This area applies predictive knowledge to determine degradation patterns that precede equipment failures and to predict the remaining system performance after component failures.

- Planning and Scheduling: This area applies apportioning knowledge to allocate the electric power resource.

- Integration of Knowledge-based and Conventional Automation: This area applies problem-solving knowledge to coordinate and focus both algorithmic and knowledge-based problem solving activities.

To develop technology for these knowledge areas, we have embarked on an aggressive prototype design and demonstration program. The project is jointly funded by NASA's Office of Aeronautics and Space Technology and NASA's Office of Space Station Freedom.

\subsection{Automation Architecture}

Because electrical power system management is a mature terrestrial discipline, tried and true operating philosophies and techniques exist that can be applied directly to Space Station Freedom. We believe that this is prudent and have defined Freedom's power system's operation using electric utility practices and procedures as the motif. In the terrestrial power systems, algorithmic decision aids are used by experienced dispatchers to guide their command and control considerations [1]. We propose to augment this approach by developing expert systems to direct the analyses and to integrate the results into oversight decisions. The gist is to perform a closed-loop command and control function using several specialized expert systems to perform diagnosis, security analysis, and overall coordination; and to use conventional algorithms for power scheduling. We also include a paraphrase of the Oper- ations Management Application software (OMA) ${ }^{1}$ to investigate interactions within the hierarchical architecture proposed for Freedom's baseline design. To develop and demonstrate our automation design we will use the Lewis Space Station Freedom Electric Power Test-bed and real-time simulations.

The command and control cycle, shown in Figure 1, begins with a sample of data from the electric power simulation. This data is processed by expert systems that recognize and classify the operating state of the power system and then proceed to perform specialized tasks based upon the results of the classification cycle. These expert systems perform two basic functions: 1.) system security monitoring and analysis and 2.) failure cause diagnosis.

The security monitoring and analysis software assesses the current operating state of the power system and analyzes the overload risk from possible failure modes. The system is judged secure if there are no contingencies that result in an emergency situation. Otherwise, insecure operating conditions are ranked in decreasing order of consequences and translated into constraints upon the scheduling and distribution of power.

The diagnosis software determines the most likely cause of abnormal operation. Like the security analysis software, it too generates constraints upon the scheduling and distribution of electric power.

Imperatives and correlative data are provided from the Operations Management Application software. This communique consists of the strategic commands for power system operation and pertinent global information. Since we have proposed a hierarchical, cooperative, problem-solving environment, this data is part of a bargaining transaction among the Station's resource consumers, their suppliers, and the operations executive--not unilateral commands directed to lowlevel power control functions. This emphasizes the OMA's role as a manager and coordinator and minimizes its role as an autocrat. These OMA requests are translated into constraints on scheduling and overall operation.

The Arbiter expert system software coordinates the Operations Management Application software's requests, security analysis results, and diagnostic conclusions by specifying appropriate system operating constraints and electrical loads to a scheduling algorithm. The Arbiter software also determines which schedule and operating plan is to be used given the current state of the power system's operation. This current plan is used to operate the electric power sys-

\footnotetext{
${ }^{1}$ The OMA software-part of the computerized Operations Management System-performs the automated coordination of Freedom's systems and payloads [3].
} 


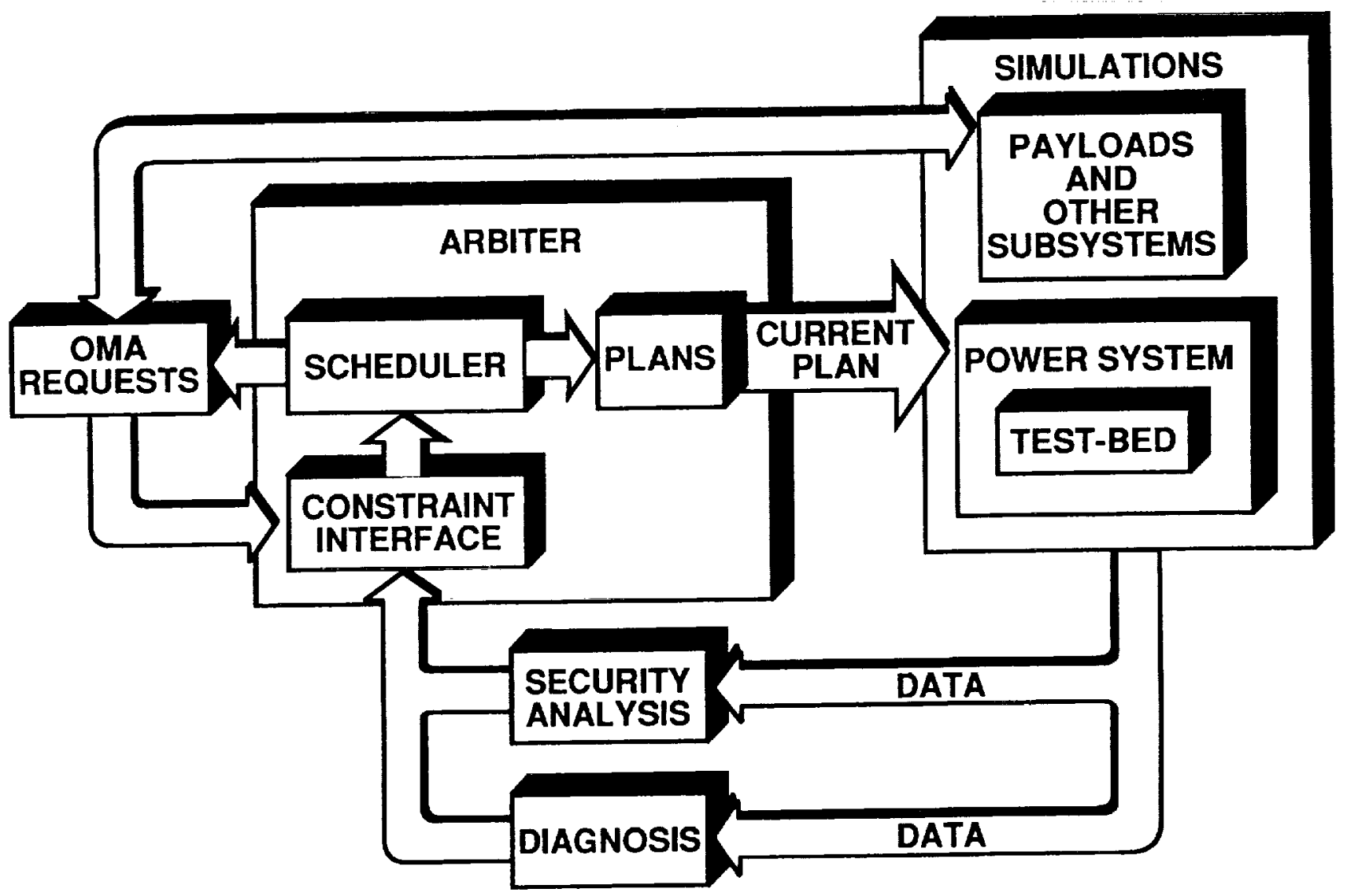

Figure 1: Automation Architecture

tem simulation.

The Scheduler software finds power profiles that maximize productivity and that satisfy the operating constraints stipulated by the Arbiter expert system. The resulting power dispatching schedules repose in memory (plans) awaiting selection by the arbitration software.

\section{Design and Development}

\subsection{Software for Security Monitoring and Analysis}

Many competent algorithms exist for the monitoring and analysis of operating security. The monitoring task consists of taking measured values of power at a few critical locations and taking the distribution network's topology to calculate all remaining voltages and currents. These parameters are then compared to the tolerances for line over-current, over-voltage, and under- voltage to produce a classification of either normal or emergency operation. The security analy- sis task is similar. Known transmission line, generator, and load disturbances are superimposed upon the current operating conditions and analyzed to predict operating tolerances for contingencies [1]. The workhorse tool for the monitoring and analysis is the fast, decoupled load-flow algorithm [4] which is used to solve for the voltages, currents and operating margins. We are concerned that the conventional approach to security monitoring and analysis will impose an extreme computational work load upon Freedom's power control computer-so much so that our power system will forgo security monitoring and analysis. Our only initiative has been with Case Western Reserve University in 1988 to apply artificial neural networks to the security monitoring task. The results are encouraging. A competent security monitor using artificial neural networks can "recall" the same operating margins as calculated by fast, decoupled load- flow in about $\frac{1}{10,000}$ of the processing time [5]. 


\subsection{Software for Diagnosis}

Two expert systems are being developed for the diagnosis function:

The first, APEX, has been developed in $\mathrm{KEE}^{2}$ for use with $20 \mathrm{kHz}$. switchgear. It is a rule-based expert system that uses antecedent driven logic for generating failure hypotheses (fault detection) and consequent driven logic for deducing the most likely hypothesis (fault isolation). An explanation facility is used to justify the failure cause. The APEX software can accommodate both static and temporal data. Temporal data is used to identify incipient failures, the detection of which is based on linear regression and correlation analysis. The algorithm finds "soft" failures by detecting graceful degradation in system performance and augments this with rules which isolate the cause of the degradation. APEX is thus capable of detecting anomalies such as: insulation breakdown in transformers, contact depletion in mechanical switches, and thermal conductivity degradation in power semiconductors. The APEX software also provides postdiagnostic recommendations, i.e., actions needed to recover from a particular type of failure $[6,8]$.

Several interfaces have been added to the APEX software: a graphic data display provides a human interface, a test-bed data acquisition interface and a power scheduler interface provide access to their respective systems. The APEX system is now able to replan power distribution after diagnosing the failure cause on its power system test-bed. Pertinent data values from the test-bed hardware are asserted into APEX's knowledge base. For the incipient fault detection, the values are stored in a first-in first-out table that contains the last 200 values for each test-bed measurement. The automated scheduler produces an optimum load profile and activates the antecedent driven failure detection to find deviations from the optimum plan [7].

The second diagnostic expert system, TROUBLE III, is being developed in $\mathrm{ART}^{3}$ for use with the photovoltaic generation and nickel-hydrogen battery storage systems. It is an expert system that uses set-covering rather than a series of if-then rules to encode the failure knowledge [9]. In this software, a data base linking all known system failures to their known symptoms is built and searched to generate the failure cause hypotheses for observed symptoms. Antecedent driven rules control hypothesis generation and determine the most likely cause. Nonmonatonic inference is implemented using reasoned assumptions [10], and rule conflicts are identified and resolved using Petri net tran-

${ }^{2}$ (C) IntelliCorp, Inc.

${ }^{3}$ (CInference Corp. sitions [11, 12]. The failure knowledge, however, is stored as data and is easily maintained. TROUBLE III uses a standard reliability analysis tool-the failure modes and effects analysis - to produce the symptom and failure data base [13]. Symptoms are detected using rule-based classifiers which process static system measurements.

\subsection{The Arbiter}

\subsubsection{Software for Coordination}

The coordination of the diagnostic, scheduling, and plan execution tasks is implemented in the Arbiter expert system. This software must make the power system's tactical operating decisions. For example, it must take a failure's cause from the diagnosis soft ware; search the list of plans to find whether or not one exists for this contingency and implement it. If there is no contingency plan, then Arbiter must estimate the failure's impact on power generation, storage, or distribution and negotiate through the OMA with the power consumers to prepare a new operating plan. To maximize secure operations, it must prepare contingency plans (by negotiating with the consumers) for each of the risky failure modes evaluated with the security analysis software (Section 3.1). Our development plan for the Arbiter software is to bring automated security monitoring and analysis, failure-cause diagnosis, and scheduling products together in a rapid prototyping environment; to discover through experimentation with these products cardinal decision situations and their corresponding tactics; and to capture this situation-response knowledge in an expert system.

\subsubsection{Software for Scheduling}

Automating Freedom's resource apportioning task (Section 2.1) is most challenging. To achieve an acceptable allocation of power requires cooperative problem- solving among subsystems, payloads, and the Operations Management Application software to maximize productivity. The decisions that are made to schedule power must take into account the preferences of the power users, the constraints of the power system operation, and the overall productivity of the power schedule. We have been experimenting with a concept for interaction among the OMA, the subsystems, and the payloads which will produce decentralized, automated resource scheduling and which will reduce information transfer.

Space Station Freedom's functional agents can be organized into three categories: 1.) the operations management agent-the top-level project manager, 2.) resource managing agents--those which supply basic 
resources throughout the station, and 3.) resource requesting agents - those who plan to use the resources to perform other functions. With our concept, each agent is allowed to make all the decisions about controlling any of the activities under his purview. Resource requesting agents will plan activities according to what is considered an acceptable discharge of their functional responsibility. These activities, in turn, will require resources to be supplied by the resource managing agents who must organize to support the activities of all of the consumers. This conceptualization yields the following desiderata:

1. A two-level hierarchy. Decision-making agents organized by function who either plan what is to be done or who manage the required resources constitute the bottom level. An operations management agent who integrates the decisions and resolves deadlocks constitutes the top level.

2. Explicit value systems. Decision-making agents use cardinal measures of preference for trade-offs among different resource consumption tasks to maximize station productivity and to preserve equity among the consumers.

3. Local constraints. Resource supplying agents schedule demands using only the constraints in their own subsystem. Resource requesting agents plan activities that do not violate any of their operating constraints.

To automate these distributed activities, scheduling software is being developed which uses a freemarket economic model to coordinate the bidding for resources. This free-market model provides a timedependent pricing mechanism that ensures a balance between supply and demand. First, the operations management agent acting as a free-market coordinator postulates trial prices for all resources and establishes a group value function for each consumer. Next, the resource consumers produce a time profile of resource consumption that maximizes the difference between their task's value and its resource costs. Finally, the resource managing agents poll the requesters, establish an integrated usage profile, and quote the fair price they would charge to supply this demand. The free-market coordinator compares the fair prices with the initial trial prices and establishes a new set of trial prices. The bidding cycle is repeated until the fair price quoted by the resource supplying agents matches the trial price [14].

\subsubsection{Software for Battery Management}

A battery management expert system is being designed which will extend battery life without sacrificing load scheduling flexibility. This system will perform life prediction and state-of-charge estimation by compiling and analyzing trends in battery data. Using this information, the battery management software will synthesize charging and discharging power versus time profiles that maximize battery life. The preferred charging and discharging profiles will be used by the Arbiter coordination software (Section 3.3.1) during the interactive resource scheduling described in Section 3.3.2. The Arbiter will adjust the fair price based upon the battery's life as well as upon its charge.

\subsection{Test-beds and Simulations}

Two development paths are being pursued: The first uses the APEX switchgear diagnostic system and a zero-one, constrained optimization scheduling algorithm to produce load shedding or reconfiguration commands for a small $20 \mathrm{kHZ}$ test-bed. This test-bed (known as the $20 \mathrm{kHz}$ Brass Board) contains several pieces of switchgear and a network of microprocessors for gathering data and commanding the switchgear [8]. The objectives are: to demonstrate switchgear failure detection and diagnosis, to explore cooperative problem solving between planning and diagnostic systems, and to determine the difficulty of integrating a LISP-based development computer with an Ada-implemented distributed control system. This information will be used to guide the integration of our automation products into the Space Station Freedom Power System Test-bed.

The second development path uses simulations of the resource consumers and producers to create an experimental environment in which to bargain for resources. Enough payloads and resource producing subsystems will be modeled to identify the intensity of the communications. We intend to incorporate the Space Station Freedom Electric Power Test-bed into the simulation environment to verify that the automation designs are competent for real hardware. This corroboration of simulation-based findings will use as much power system hardware and software as practicable.

\section{Postlude}

The high cost of the initial space station is a major issue. A strong justification for any new technological alternative is needed if it is to secure a berth aboard Freedom [2]. Our objective is to design a competent, highly-automated electric power system that will 
quickly amortize its development expense and yield the dividend of increased productivity for the balance of Freedom's life. We believe that prudently integrating expert systems with conventional algorithms will significantly reduce Freedom's operating costs and that this benefit warrants our investigations. Our approach will reduce the ground crew needed to manage power system operation, reduce the station crew's time used to support ground-based command and control activities, and will reduce the amount of extravehicular-activity required for maintenance and repair.

Our first challenge is to develop a prototype. Second, we must determine our prototype's effectiveness as an alternative to Freedom's baseline design. Finally, we must ascertain its development costs. We will begin by:

1. Identifying "hooks" and "scars" for automation application and growth.

2. Substantiating the readiness of knowledge-based automation technologies for application aboard Space Station Freedom.

3. Defining the solution for cooperative problemsolving among the power system, other resource producing subsystems, OMA, and payloads.

Only through concerted evaluations will we provide the rationale for flying a productive, high technology power system aboard Space Station Freedom.

\section{References}

[1] J. L. Dolce, "An integrated approach to space station power system autonomous control," in Proc. 22nd Intersociety Energy Conversion Engineering Conference, AIAA 1, 499-508 (1987).

[2] J. L. Dolce and K. A. Faymon, "Automating the U.S. Space Station's electrical power system," in Optical Engineering, 25(11), 1181-1185 (Nov. 1986).

[3] OMS Evolution Plan, NASA, Space Station Freedom Program, OMS Evolution Team, unpublished (Oct. 25, 1988).

[4] B. Stott and O. Alsac, "Fast decoupled load flow," in Transactions on Power Apparatus and Systems, IEEE, PAS-93, 859-869 (May 1974).

[5] J. L. Dolce, D. J. Sobajic, and Y. Pao, "Automating security monitoring and analysis for Space Station Freedom's electric power system," in Proc. 25th Intersociety Energy Conversion Engineering Conference, AIChE (1990).
[6] L. V. Truong, J. L. Walters, M. E. Roth, T. Quinn, and W. M. Krawczonek, "Autonomous power expert fault diagnostic system for Space Station Freedom electrical power system testbed," in Proc. Third Annual Space Operations Automation and Robotics, NASA, CP-3059, (July 1989).

[7] J. L. Walters, E. J. Petrik, M. E. Roth, L. V. Truong, T. Quinn, and W. M. Krawczonek, "Autonomous power expert system," 1990 Goddard Conference on Space Applications of Artificial Intelligence, NASA, Goddard Space Flight Center, Greenbelt, MD. (May 1990).

[8] M. Ringer and T. Quinn, "Autonomous power expert system (APEX)," in Proc. 25th Intersociety Energy Conversion Engineering Conference, AIChE (1990).

[9] L. M. Fesq and A. Stephan, "Advances in spacecraft autonomy using artificial intelligence techniques," in Proc. 12th Annual AAS Guidance and Control Conference, AAS, Keystone, CO. (Feb. $4-8,1989)$.

[10] J. Doyle, Some Theories of Reasoned Assumptions: An essay in rational psychology, CarnegieMellon University, Dept. of Computer Science, CMU-83-125, Pittsburgh, PA., (1983).

[11] A. W. Holt, "Introduction to occurrence systems," in Associative Information Techniques, E. L. Jacks, ed., American Elsevier, New York (1971).

[12] M. D. Zisman, "Use of production systems for modeling asynchronous, concurrent processes," in Pattern-Directed Inference Systems, D. A. Waterman and F. Hayes-Roth, eds., Academic Press, New York, (1978).

[13] J. L. Dolce and K. A. Faymon, "A systems engineering approach to automated failure cause diagnosis in space power systems," in Proc. 22nd Intersociety Energy Conversion Engineering Conference, AIAA 1, 590-600 (1987).

[14] P. A. Mellor, J. L. Dolce, and J. C. Krupp, "Electric power scheduling: a distributed problemsolving approach for Space Station Freedom," in Proc. 25th Intersociety Energy Conversion Engineering Conference, AIChE (1990). 


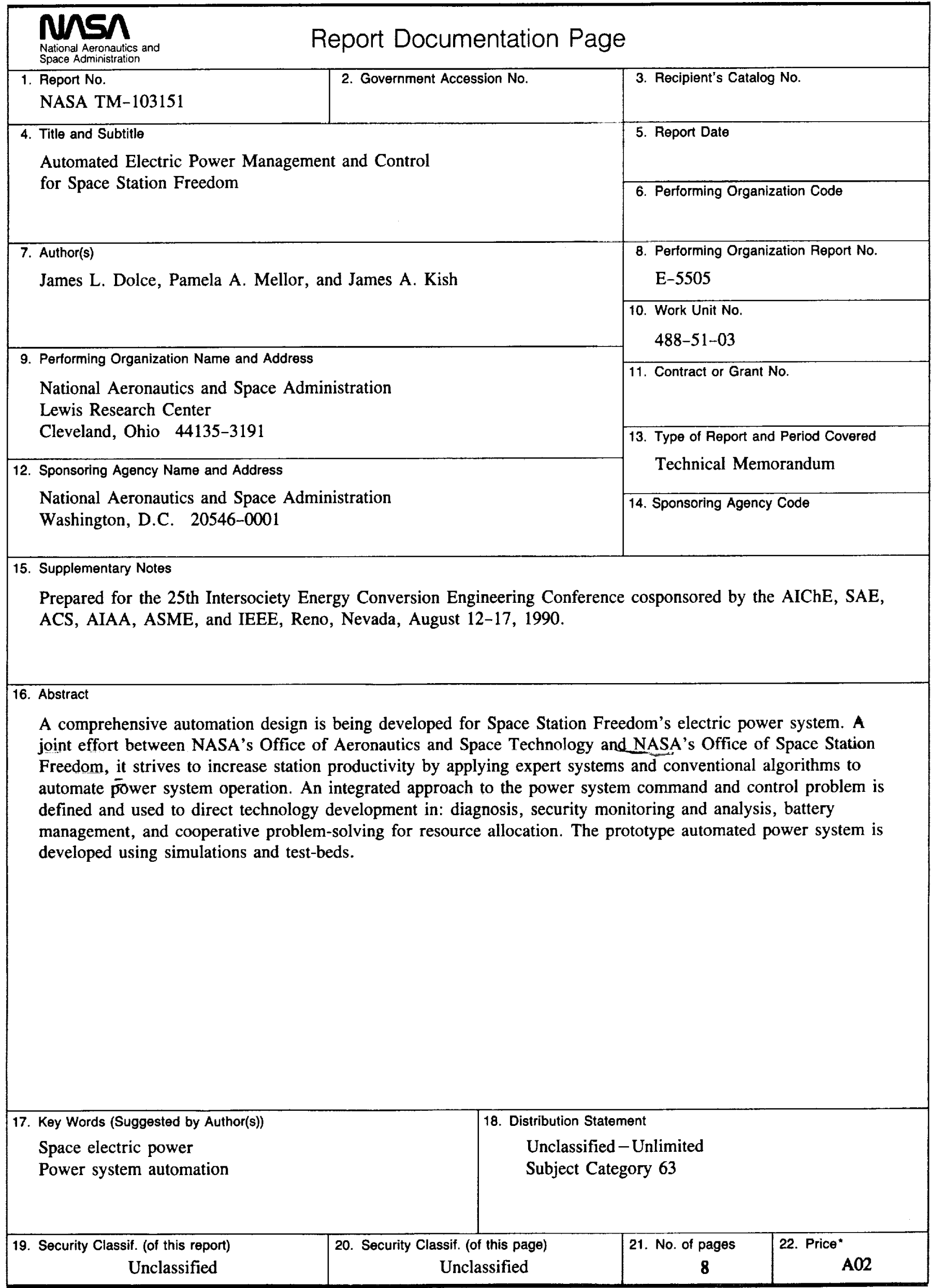


